\title{
Bulk-mediated Surface Diffusion on a Cylinder in the Fast Exchange Limit
}

\author{
A. V. Chechkin ${ }^{1}$, I. M. Zaid ${ }^{2}$, M. A. Lomholt ${ }^{3}$, I. M. Sokolov ${ }^{4}$, R. Metzler ${ }^{5} *$ \\ ${ }^{1}$ Institute for Theoretical Physics NSC KIPT, Akademicheskaya st.1, 61108 Kharkov, Ukraine and \\ Max-Planck Institute for Physics of Complex Systems, Nöthnitzer Str. 38, D-01187 Dresden, FRG \\ ${ }^{2}$ Rudolf Peierls Centre for Theoretical Physics, University of Oxford, 1 Keble Road, Oxford OX1 3NP, \\ United Kingdom \\ ${ }^{3}$ MEMPHYS - Center for Biomembrane Physics, Department of Physics, Chemistry, and Pharmacy, \\ University of Southern Denmark, Campusvej 55, DK-5230 Odense M, Denmark \\ ${ }^{4}$ Institut für Physik, Humboldt Universität zu Berlin, Newtonstraße 15, D-12489 Berlin, FRG \\ ${ }^{5}$ Institute for Physics \& Astronomy, University of Potsdam, D-14476 Potsdam-Golm, Germany and \\ Department of Physics, Technical University of Tampere, FI-33101 Tampere, Finland
}

\begin{abstract}
In various biological systems and small scale technological applications particles transiently bind to a cylindrical surface. Upon unbinding the particles diffuse in the vicinal bulk before rebinding to the surface. Such bulk-mediated excursions give rise to an effective surface translation, for which we here derive and discuss the dynamic equations, including additional surface diffusion. We discuss the time evolution of the number of surface-bound particles, the effective surface mean squared displacement, and the surface propagator. In particular, we observe sub- and superdiffusive regimes. A plateau of the surface mean-squared displacement reflects a stalling of the surface diffusion at longer times. Finally, the corresponding first passage problem for the cylindrical geometry is analysed.
\end{abstract}

Keywords and phrases: Bulk-mediated diffusion, anomalous diffusion, Lévy flights, stochastic processes

Mathematics Subject Classification: 82C41, 82C70, 60G15, 60G20

\section{Introduction}

The process of bulk mediated surface diffusion (BMSD) shown in Fig. 1 describes the stochastic motion of particles that transiently adsorb to a physical surface, unbind to perform a diffusive path in the bulk volume adjacent to the surface, before eventually rebinding to the surface. Additionally the particles may diffuse along the surface. This intermittent behaviour is characterised by the diffusivities $D_{b}$ in the three-dimensional bulk and $D_{s}$ along the two-dimensional surface. The repeated bulk excursions effect decorrelations in the pure surface motion and thus lead to a more efficient surface exploration by the particles.

${ }^{*}$ Corresponding author. E-mail: rmetzler@uni-potsdam.de 


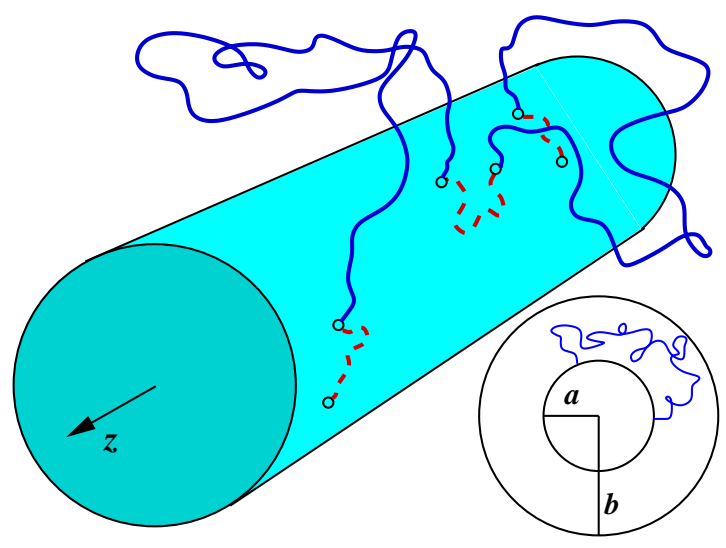

Figure 1. A particle diffuses in the bulk (full lines) and intermittently binds to a cylinder surface on which it may also diffuse (broken lines). This produces an effective surface motion. We here consider the motion along the cylinder. Bottom right: frontal of diffusion between inner and outer cylinder.

Theoretically BMSD was previously investigated for a planar surface in terms of scaling arguments [1], an adsorption barrier model [2], master equation schemes [3], Langmuir kinetics [4], and simulations studies [5]. More recent studies have been concerned with effects of bulk-surface exchange on narrow escape problems [6], on reaction rates in interfacial systems [7], and with the surface diffusion of copper atoms in nanowire fabrication [8]. The effect of bulk-mediated excursions of reactive species on the longtime behavior of the catalytic reactions in systems with a catalytic surface is in contact with a liquid phase, was studied in Ref. [9].

Experimentally, BMSD has been measured by nuclear magnetic resonance (NMR) methods. NMR is sensitive to the preferred orientation of adsorbed molecules on the surface of materials. On local pore surfaces the non-planar geometry produces spin reorientations for particles diffusing on the surface [10]. In addition BMSD excursions and the ensuing Lévy walk-like superdiffusion can indeed by resolved by NMR [11]. NMR methods were also used to measure the effective surface diffusion on cylindrical mineralic rods [12]. Note that BMSD on cylindrical surfaces is also relevant for the transient binding of chemicals to nanotubes [13], and for numerous other technological applications [5].

Biologically, BMSD along a cylinder occurs in the context of gene regulation. Thus DNA-binding proteins may diffuse three-dimensionally in the bulk, in which the DNA is embedded, or transiently bind to the DNA surface and diffusively slide along the cylinder [14-16]. Similarly, while molecular motors move actively along cylindrical filaments of the cellular skeleton, they regularly fall off the filaments and perform bulk mediated motion [17]. Finally, BMSD in cylindrical geometries occurs during the surface exchange of molecules with the vicinal bulk solution in rod-shaped cells such as bacilli and linear arrangements thereof [18].

Following our previous studies [19] we here summarise an exact approach to BMSD for a reactive cylindrical surface in terms of diffusion equations for the surface and bulk densities of particles which are coupled through an adsorbing current. We derive and discuss explicit results for the number of particles occupying the cylindrical surface, the effective mean squared displacement (MSD) along the surface, and the returning time distribution from the bulk to the cylinder. Within this approach different dynamic regimes naturally arise from the physical time scales entering our description. Thus, at shorter times we derive the famed superdiffusive spreading with time of the surface MSD, $\left\langle z^{2}(t)\right\rangle \simeq t^{3 / 2}[1]$, as well as the associated Cauchy form of the surface probability density of particles as well as its cutoff. At longer times we surprisingly observe that the surface MSD $\left\langle z^{2}(t)\right\rangle$ turns over to a plateau, reflecting a tradeoff between the increasing number of particles leaving the surface and the ever increasing distance along the 
cylinder covered in longer and longer bulk excursions. When the system is confined spatially, this plateau eventually turns into normal diffusion.

\section{Coupled diffusion equations and general solution}

In this Section we establish the starting equations for BMSD diffusion on a cylindrical surface with azimuthal symmetry and present the general solution in Fourier-Laplace space. In what follows we assume that the inner cylinder may be surrounded, concentrically, by a larger cylinder with reflecting boundaries (see, Fig. 1).

\subsection{Starting equations and particle number conservation}

Our geometry is specified by the coordinates $z$ along the cylinder axis, the radius $r$ perpendicular to the $z$ axis, and the azimuthal angle $\theta$ between the radius and the $x$ axis. As we are only interested in the effective particle displacement along the $z$ axis we eliminate the $\theta$ dependence by considering a smeared-out initial condition and boundary condition for the cylinder surface. Thus both the bulk and the surface particle concentration functions become independent of the angle $\theta$. We can then describe the process by the bulk concentration $\mathcal{C}=\mathcal{C}(r, z, t)$ of physical dimension $[\mathcal{C}]=1 / \mathrm{cm}^{3}$ and the surface concentration $n_{2 \mathrm{D}}(z, t)$ with $\left[n_{2 \mathrm{D}}\right]=1 / \mathrm{cm}^{2}$. Averaging this cylinder surface density over the azimuthal angle, obtaining the line density $n(z, t)$ :

$$
n(z, t)=2 \pi a n_{2 \mathrm{D}}(z, t),
$$

such that $[n]=1 / \mathrm{cm}$. The factor $2 \pi a$ becomes relevant when we specify the reactive (sometimes also referred to as radiative or Robin) boundary condition on the inner cylinder connecting surface line density $n(z, t)$ and bulk density $\mathcal{C}(r, z, t)$.

Based on the quantity $n(z, t)$, the total number $N_{s}(t)$ of surface particles becomes

$$
N_{s}(t)=2 \pi a \int_{-\infty}^{\infty} n_{2 \mathrm{D}}(z, t) d z=\int_{-\infty}^{\infty} n(z, t) d z
$$

We assume that initially $N_{0}$ particles are concentrated in a $\delta$-peak on the cylinder surface at $z=0$,

$$
\left.n(z, t)\right|_{t=0}=N_{0} \delta(z)
$$

Thus the initial bulk concentration vanishes everywhere on the interval $a<r \leq b$ such that

$$
\left.\mathcal{C}(r, z, t)\right|_{t=0}=0
$$

The boundary conditions are specified as follows. At the outer cylinder $(r=b)$ we impose the reflecting boundary condition

$$
\left.\frac{\partial}{\partial r} \mathcal{C}(r, z, t)\right|_{r=b}=0
$$

When no outer cylinder is present (i.e., $b \rightarrow \infty$ ), this von Neumann condition is replaced by a natural boundary condition of the form

$$
\lim _{r \rightarrow \infty} \mathcal{C}(r, z, t)=0 .
$$

As mentioned above, on the inner cylinder $(r=a)$ we encounter a reactive boundary condition. We derive this condition from a discrete random walk in Refs. [19]. Thus, we balance the flux away from the inner cylinder surface,

$$
j_{\text {off }}=\frac{1}{\tau_{\text {off }}} n_{2 \mathrm{D}}(z, t)=\frac{1}{2 \pi a \tau_{\text {off }}} n(z, t),
$$


by the incoming flux from the bulk onto the cylinder surface,

$$
j_{\text {on }}=\lim _{r \rightarrow a} k_{b} \mathcal{C}(r, z, t) .
$$

Here, the characteristic time scale for unbinding from the surface, $\tau_{\text {off }}$ has physical dimension $\left[\tau_{\text {off }}\right]=$ sec. $\tau_{\text {off }}$ is proportional to the Arrhenius factor of the binding free energy $\varepsilon$ of the particles, $\exp \left(|\varepsilon| /\left[k_{B} T\right]\right)$, where $k_{B} T$ denotes thermal energy at temperature $T$. The binding rate $k_{b}$ has dimension $\left[k_{b}\right]=\mathrm{cm} / \mathrm{sec}$, characteristic for surface-bulk coupling in cylindrical coordinates $[14,20]$. These parameters define the coupling constant

$$
\mu \equiv \frac{1}{2 \pi a k_{b} \tau_{\mathrm{off}}},
$$

such that our reactive boundary condition becomes

$$
\left.\mathcal{C}(r, z, t)\right|_{r=a}=\mu n(z, t)
$$

The dynamic equation for the bulk density $\mathcal{C}(r, z, t)$ is given by the cylindrical diffusion equation

$$
\frac{\partial}{\partial t} \mathcal{C}(r, z, t)=D_{b}\left(\frac{1}{r} \frac{\partial}{\partial r}\left[r \frac{\partial}{\partial r}\right]+\frac{\partial^{2}}{\partial z^{2}}\right) \mathcal{C}(r, z, t),
$$

valid on the domain $a<r<b$ and $-\infty<z<\infty$. In Eq. (2.11), $D_{b}$ is the bulk diffusion coefficient of dimension $\left[D_{b}\right]=\mathrm{cm}^{2} / \mathrm{sec}$. The dynamics of the bulk density couples to that of the line density $n(z, t)$ through the surface flux terms in the following form [19]

$$
\frac{\partial}{\partial t} n=D_{s} \frac{\partial^{2}}{\partial z^{2}} n(z, t)+\left.2 \pi a D_{b} \frac{\partial}{\partial r} \mathcal{C}(r, z, t)\right|_{r=a}
$$

where $D_{s}$ denotes the surface diffusion coefficient. In many realistic cases the magnitude of $D_{s}$ is considerably smaller than the bulk diffusivity $D_{b}[16,21]$. We note that our starting equations $(2.11)$ and $(2.12)$ supplemented with the boundary condition (2.10) are exactly the equations of Langmuir kinetics used in Refs. [4] to study proton diffusion from a source to a detector on the membrane surface, in the regime of fast exchange between the membrane surface and the bulk solution. Equations (2.11) to (2.12) imply that that the bulk-mediated, effective surface diffusion evolves under the condition of instantaneous equilibrium between the surface concentration and that of the immediately adjacent bulk layer, such that at each moment of time both concentrations are proportional [4]. The condition for the involved characteristic time scales necessary for instantaneous equilibrium is formulated below, see Section II B. In what follows we demonstrate that the fast exchange regime exhibits interesting anomalous behaviour, including the famed superdiffusive effective surface diffusion originally discovered in Ref. [1].

\subsection{Solution of starting equations}

Equation (2.12) and the corresponding initial and boundary value problem is solved in Fourier-Laplace space. The respective transforms are defined in terms of

$$
n(k, t)=\mathscr{F}\{n(z, t) ; z \rightarrow k\}=\int_{-\infty}^{\infty} e^{i k z} n(z, t) d z,
$$

and

$$
n(z, s)=\mathscr{L}\{n(z, t) ; t \rightarrow s\}=\int_{0}^{\infty} e^{-s t} n(z, t) d t .
$$

Here and in the following we express the transform of a function by explicit dependence on the Fourier or Laplace variable, thus, $n(k, s)$ is the Fourier-Laplace transform of $n(z, t)$. 
The details of the solution procedure are documented in Refs. [19], and we find for the surface density in the Fourier-Laplace domain that

$$
n(k, s)=\frac{N_{0}}{s+k^{2} D_{s}+\kappa q \frac{\Delta_{1}(k, s)}{\Delta(k, s)}},
$$

where the coupling constant

$$
\kappa \equiv 2 \pi a \mu D_{b}=\frac{D_{b}}{k_{b} \tau_{\text {off }}}
$$

is of physical dimension $[\kappa]=\mathrm{cm} / \mathrm{sec}$ and serves as a measure of the efficiency of the surface-bulk exchange. Moreover, we use the abbreviation $q^{2}=k^{2}+s / D_{b}$. With the help of $\kappa$, we distinguish the regimes of strong, intermediate, and weak bulk-surface coupling used in this work. In particular, the limit $\kappa \rightarrow 0$ corresponds to vanishing coupling such that the surface propagator (2.14) represents the standard Gaussian propagator of surface-confined, normal diffusion. The functions $\Delta$ and $\Delta_{1}$ in Eq. (2.14) contain the modified Bessel functions $I_{\nu}$ and $K_{\nu}$,

$$
\begin{aligned}
\Delta(k, s) & =I_{0}(q a) K_{1}(q b)+K_{0}(q a) I_{1}(q b), \\
\Delta_{1}(k, s) & =K_{1}(q a) I_{1}(q b)-I_{1}(q a) K_{1}(q b) .
\end{aligned}
$$

Finally, from the Fourier-Laplace transform (2.14) the number of particles on the cylinder surface amounts to

$$
N_{s}(s)=\lim _{k \rightarrow 0} n(k, s),
$$

following the properties of the Fourier transform.

Based on the surface propagator $n(z, t)$, Eq. (2.14), we obtain the single-particle mean squared displacement for the effective surface diffusion on the cylinder,

$$
\left\langle z^{2}(t)\right\rangle=\frac{1}{N_{0}} \int_{-\infty}^{\infty} z^{2} n(z, t) d z
$$

In Fourier-Laplace domain, this quantity becomes

$$
\left\langle z^{2}(s)\right\rangle=-\left.\frac{1}{N_{0}} \frac{\partial^{2} n(k, s)}{\partial k^{2}}\right|_{k=0} .
$$

This is the mean squared displacement for particles, that are present on the surface at the time instant $t$. This displacement effectively slows down since the concentration $N_{s}(t)$ of surface particles decreases with time. If we want to exclude the effect of the concentration decrease, we may introduce the normalised mean squared surface displacement

$$
\left\langle z^{2}(t)\right\rangle_{\text {norm }}=\frac{N_{0}}{N_{s}(t)}\left\langle z^{2}(t)\right\rangle
$$

. In general, we observe that $\left\langle z^{2}(t)\right\rangle_{\text {norm }} \geq\left\langle z^{2}(t)\right\rangle$.

Inspection of the solution (2.14) reveals three typical time scales apparent in the problem. Namely, the first is the characteristic coupling time

$$
t_{\kappa} \equiv \frac{D_{b}}{\kappa^{2}}=\frac{k_{b}^{2} \tau_{\text {off }}^{2}}{D_{b}}
$$

When the coupling between bulk and cylinder surface is weak, $\kappa \rightarrow 0$, the corresponding coupling time $t_{\kappa}$ diverges. It vanishes in the opposite case when the coupling is strong, $\kappa \rightarrow \infty$. While the time scale 
$t_{\kappa}$ is characteristic of the bulk-surface exchange, the geometry of the problem imposes the two remaining characteristic times: the inner and outer cylinder radii imply the time scales

$$
t_{a} \equiv \frac{a^{2}}{D_{b}}
$$

and

$$
t_{b} \equiv \frac{b^{2}}{D_{b}},
$$

respectively. By definition, $t_{b}>t_{a}$. At times shorter than $t_{a}$ a diffusing particle behaves as if it were facing a flat surface, while at times longer than $t_{a}$ it senses the cylindrical shape of the surface (note the initial placement of particles on the surface). Similarly, $t_{b}$ defines the scale when a particle starts to engage with the outer cylinder and therefore senses the confinement.

From the characteristic times $t_{\kappa}, t_{a}$, and $t_{b}$ we construct three distinct regimes, (i) the strong coupling limit

$$
t_{\kappa} \ll t_{a} \ll t_{b},
$$

where the shortest time scale is the coupling time. This regime is the most interesting as it leads to the transient Lévy walk-like superdiffusive behaviour. We note that the necessary condition for instantaneous equilibrium, $j_{\text {on }}=j_{\text {off }}$ (see Eqs. (7) and (8)) requires $t_{\kappa} \gg \tau_{\text {off }}$, see Ref. [22]. (ii) Second, we obtain the intermediate coupling limit

$$
t_{a} \ll t_{\kappa} \ll t_{b}
$$

here the superdiffusive regime is considerably shorter, however, an interesting transition regime is observed. (iii) Finally, we have the weak coupling limit

$$
t_{a} \ll t_{b} \ll t_{\kappa} .
$$

To limit the scope of this paper we will not consider this latter case in the following. However, note that when $t \ll t_{b}$ the behaviour will be similar to the $t \ll t_{\kappa}$ part of the intermediate coupling limit (ii).

\section{Number of surface particles and mean squared surface displacement}

In this Section we summarise the results for the number of surface particles and mean squared surface displacement in table form for the strong and intermediate coupling regimes.

\subsection{Strong coupling limit}

The number of particles on the surface turns from an initially constant behaviour to an inverse square root decay once the particles engage into surface-bulk exchange. At longer times, the escape of particles to the bulk becomes faster and follows a $1 / t$ law. Eventually the confinement by the outer cylinder comes into play, and we reach a stationary limit. It is the the mean squared surface displacement, for which we observe a number of very interesting behaviours. One is the initial, anomalously diffusive evolution $\simeq t^{3 / 2}[23]$. This superdiffusion is effected by bulk excursion mediation resulting in the effective Cauchy distribution

$$
n(z, t) \sim \frac{N_{0} \kappa t}{\pi\left(z^{2}+\kappa^{2} t^{2}\right)},
$$

see the next Section. In this initial regime we may come up with a simple scaling argument for the superdiffusive behaviour, compare the discussion in Ref. [1]. Accordingly, once detached from the surface, a particle returns to the surface with a probability distributed according to $\simeq t^{-1 / 2}$. Due to the diffusive coupling $z^{2} \simeq t$ while in the bulk, the effective displacement along the cylinder is then distributed according to $\simeq|z|^{-1}$, giving rise to a probability density $\simeq z^{-2}$. The Cauchy distribution therefore arises due to this change of variables in the probability densities: for the first return we have $\wp_{\text {ret }}(t) \simeq t^{-3 / 2}$, 


\begin{tabular}{|c|c|c|c|}
\hline Time regime & $N_{s}(t)$ & $\left\langle z^{2}(t)\right\rangle$ & $\left\langle z^{2}(t)\right\rangle_{\text {norm }} \equiv \frac{\left\langle z^{2}(t)\right\rangle}{N_{s}(t) / N_{0}}$ \\
\hline$t<t_{\kappa}$ & $N_{0}\left(1-\frac{2}{\sqrt{\pi}}\left[\frac{t}{t_{\kappa}}\right]^{1 / 2}\right)$ & $2 D_{s} t\left(1+\frac{2}{3 \sqrt{\pi}} \frac{D_{b}}{D_{s}}\left[\frac{t}{t_{\kappa}}\right]^{1 / 2}\right)$ & $2 D_{s} t\left(1+\frac{2}{3 \sqrt{\pi}} \frac{D_{b}}{D_{s}}\left[\frac{t}{t_{\kappa}}\right]^{1 / 2}\right)$ \\
\hline$t_{\kappa}<t<t_{a}$ & $\sqrt{\frac{t_{\kappa}}{\pi}} \frac{N_{0}}{t^{1 / 2}}$ & $2 D_{s} t_{\kappa}+\frac{2 \sqrt{t_{\kappa}}}{\sqrt{\pi}} D_{b} t^{1 / 2}$ & $2 \sqrt{\pi t_{\kappa}} D_{s} t^{1 / 2}+2 D_{b} t$ \\
\hline$t_{a}<t<t_{b}$ & $\frac{1}{2} \sqrt{t_{a} t_{\kappa}} \frac{N_{0}}{t}$ & $t_{a} t_{\kappa} D_{s} \frac{1}{t} \ln \left(\frac{C t}{t_{a}}\right)+\sqrt{t_{a} t_{\kappa}} D_{b}$ & $2 \sqrt{t_{a} t_{\kappa}} D_{s} \ln \left(\frac{C t}{t_{a}}\right)+2 D_{b} t$ \\
\hline$t_{b}<t$ & $2 \frac{\sqrt{t_{a} t_{\kappa}}}{t_{b}} N_{0}$ & $\frac{8 t_{a} t_{\kappa}}{t_{b}^{2}} D_{s} t+\frac{4 \sqrt{t_{a} t_{\kappa}}}{t_{b}} D_{b} t$ & $4 \frac{\sqrt{t_{a} t_{\kappa}}}{t_{b}} D_{s} t+2 D_{b} t$ \\
\hline
\end{tabular}

TABLE 1. Effective surface diffusion, strong coupling limit. For the different regimes we list the number of particles $N_{s}(t)$ on the surface, the surface mean squared displacement $\left\langle z^{2}(t)\right\rangle$, and the normalised mean squared displacement $\left\langle z^{2}(t)\right\rangle_{\text {norm }} \cdot C=\exp (\gamma) \approx$ 1.78107 where $\gamma$ is Euler's constant.

and thus the surface displacement corresponds to $n(z) d z=\wp(t) d t$. With $z^{2} \simeq t$ this implies $n(z) \sim$ $\wp(t(z)) d t / d z \sim z^{-3} \times z \sim z^{-2}$.

Later, the mean squared surface displacement turns over to a square root growth in time, corresponding to subdiffusion. As can be seen from the associated normalised mean squared surface displacement, this behaviour is due to the escaping particles. At even longer times the mean squared displacement reaches a plateau value. This is a remarkable property of this cylindrical geometry, reflecting a delicate balance between the decreasing particle number and the increasing length of the bulk mediated surface translocations. This plateau is the terminal behaviour when no outer cylinder is present. That is, even at infinite times, when fewer and fewer particles are on the surface, the surface mean squared displacement does not change! In presence of the outer cylinder the mean squared displacement will eventually be dominated by the bulk motion and acquires the normal linear growth with time.

Combining the dynamics of the number of surface particles and the mean squared displacement we obtain the behaviour of the normalised mean squared displacement listed in the last column of Table 1.

Note that at times longer than $t_{\kappa}$ the normalised mean squared surface displacement grows linearly with time implying normal diffusion, however, governed by the bulk diffusion coefficient $D_{b}$, not by the surface diffusivity $D_{s}$.

\subsection{Intermediate coupling limit}

In the intermediate coupling limit the results are collected in Table 2. Also in this regime we observe the initial superdiffusion and associated Cauchy form of the surface particle concentration. The subsequent regime of intermediate times splits up into two subregimes. Interestingly, in the subregime for times $t_{a}<t<t_{c}<t_{\kappa}$ the mean squared surface displacement grows almost ballistically, with logarithmic correction: this shows how the initial, superdiffusive regime $\simeq t^{3 / 2}$ is modified due to the fact that the particles in the bulk make longer excursions since the cylindrical geometry already comes into play. The 


\begin{tabular}{|c|c|c|c|}
\hline Time regime & $N_{s}(t)$ & $\left\langle z^{2}(t)\right\rangle$ & $\left\langle z^{2}(t)\right\rangle_{\text {norm }}$ \\
\hline$t<t_{a}$ & $N_{0}$ & $2 D_{s} t\left(1+\frac{2}{3 \sqrt{\pi}} \frac{D_{b}}{D_{s}}\left[\frac{t}{t_{\kappa}}\right]^{1 / 2}\right)$ & $2 D_{s} t\left(1+\frac{2}{3 \sqrt{\pi}} \frac{D_{b}}{D_{s}}\left[\frac{t}{t_{\kappa}}\right]^{1 / 2}\right)$ \\
\hline $\begin{array}{l}t_{a}<t<t_{c}<t_{\kappa} \\
t_{a}<t_{c}<t<t_{\kappa}\end{array}$ & $\begin{array}{c}N_{0} \\
\text { transition to } \simeq 1 / t\end{array}$ & $\begin{array}{c}2 D_{s} t+\frac{2 D_{b} t^{2}}{t_{c} \ln ^{2}\left(4 t /\left[C^{2} t_{a}\right]\right)} \\
\text { transition to } \simeq \text { const }\end{array}$ & $\begin{array}{c}2 D_{s} t+\frac{2 D_{b} t^{2}}{t_{c} \ln ^{2}\left(4 t /\left[C^{2} t_{a}\right]\right)} \\
\text { transition to } \simeq t\end{array}$ \\
\hline$t_{\kappa}<t<t_{b}$ & $\frac{1}{2} \sqrt{t_{a} t_{\kappa}} \frac{N_{0}}{t}$ & $t_{a} t_{\kappa} D_{s} \frac{1}{t} \ln \left(\frac{4 t}{C t_{a}}\right)+\sqrt{t_{a} t_{\kappa}} D_{b}$ & $2 \sqrt{t_{a} t_{\kappa}} D_{s} \ln \left(\frac{4 t}{C t_{a}}\right)+2 D_{b} t$ \\
\hline$t_{b}<t$ & $\frac{2 \sqrt{t_{a} t_{\kappa}}}{t_{b}} N_{0}$ & $\frac{8 t_{a} t_{\kappa}}{t_{b}^{2}} D_{s} t+4 \frac{\sqrt{t_{a} t_{\kappa}}}{t_{b}} D_{b} t$ & $4 \frac{\sqrt{t_{a} t_{\kappa}}}{t_{b}} D_{s} t+2 D_{b} t$ \\
\hline
\end{tabular}

TABLE 2. Effective surface diffusion, intermediate coupling limit. We introduced the abbreviation $t_{c}=\sqrt{t_{a} t_{\kappa}}$.

next subregime $t_{a}<t_{c}<t<t_{\kappa}$ includes a turnover behaviour that is difficult to estimate analytically, and we refer to the numerical analysis in Refs. [19].

The behaviour of the mean squared surface displacement and its normalised counterpart are shown in Fig. 2, for explanation see the caption.

\section{Surface propagator}

Here we summarise the properties of the surface propagator in the strong coupling regime. For simplicity we neglect surface diffusion, $D_{s}=0$. In the short time limit $t<t_{\kappa}<t_{a}<t_{b}$ the surface propagator in Fourier-Laplace space reduces to the simplified form

$$
n(k, s) \sim \frac{N_{0}}{s+\kappa \sqrt{k^{2}+s / D_{b}}} .
$$

We distinguish two distinct parts of this surface density. Let us start with the central part defined by $k^{2} \gg s / D_{b}$. The corresponding limiting form of Eq. (4.1) is then given by

$$
n(k, s) \sim \frac{N_{0}}{s+\kappa|k|} .
$$

Inverse Fourier-Laplace transform leads us to the Cauchy probability density function

$$
n(z, t) \sim \frac{N_{0} \kappa t}{\pi\left(z^{2}+\kappa^{2} t^{2}\right)} .
$$

For this central part of the surface propagator we may formulate the governing fractional-order dynamic equation $[23,24]$

$$
\frac{\partial}{\partial \kappa t} n(z, t)=\frac{\partial}{\partial|z|} n(z, t)
$$



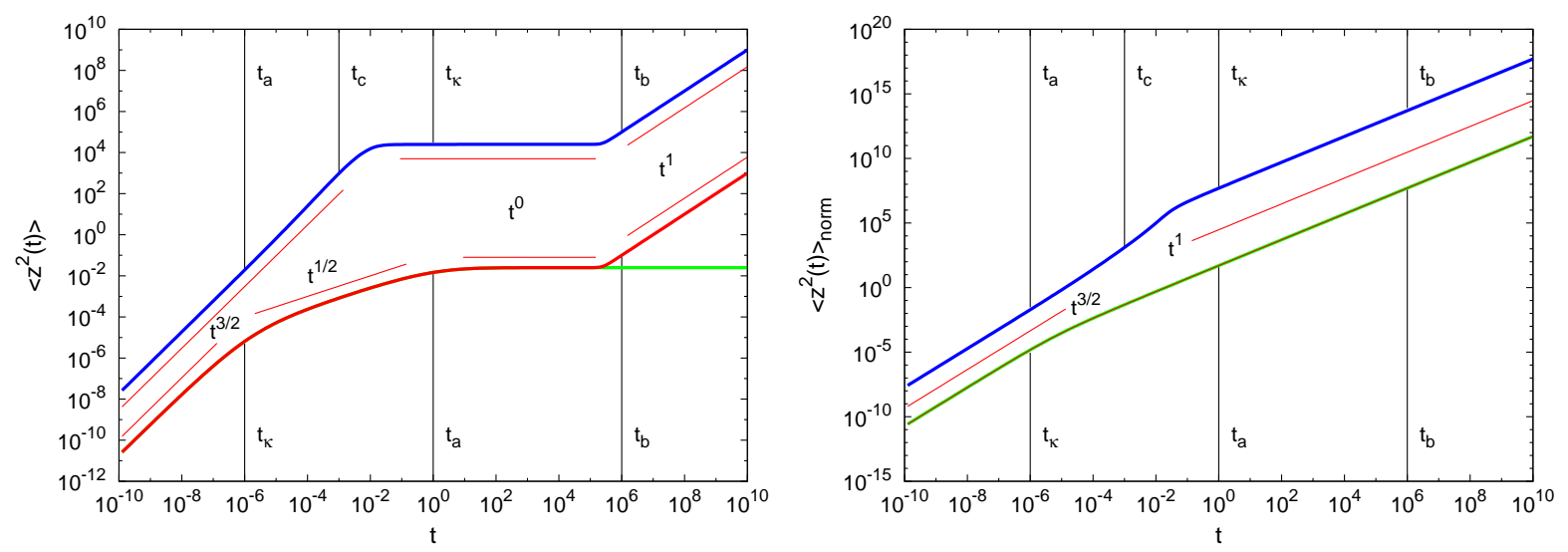

FiguRE 2. Surface mean squared displacement obtained by numerical Laplace inversion of Eqs. (55) and (56), based on Eqs. (48) and (50). Left: The graphs show the various effective diffusion regimes $\left\langle z^{2}(t)\right\rangle$ along the cylinder. Note the characteristic transient plateau, which is the terminal behavior in the absence of a confining outer cylinder. Right: Normalised surface mean squared displacement $\left\langle z^{2}(t)\right\rangle_{\text {norm }}$. The subregime $t_{c}<$ $t<t_{a}$ in the intermediate case distinctly shows a superdiffusive behavior that is even steeper than the initial $t^{3 / 2}$ scaling. We show the strong binding case with (red line) and without (green line) outer cylinder, as well as the case of intermediate binding with outer cylinder (blue line). The characteristic time scales connected with the associated curves denote the cases of strong (bottom) and intermediate (top) coupling. Parameters: (i) strong coupling case: $t_{\kappa}=10^{-6}, t_{a}=1$, and $t_{b}=10^{6}$ in dimensionless units. The bulk diffusion constant becomes $D_{b}=a^{2} / t_{a}=25$ for our choice $a=5$. Coupling constant $\kappa=a / \sqrt{t_{a} t_{\kappa}}=5 \times 10^{3}$, and outer cylinder radius is $b=a / \sqrt{t_{b} / t_{a}}=5 \times 10^{3}$. (ii) intermediate coupling case: $t_{a}=10^{-6}, t_{\kappa}=1$, and $t_{b}=10^{6}$. Bulk diffusivity to $D_{b}=25 \times 10^{6}$ and outer cylinder radius $b=5 \times 10^{6}$. These values are chosen such that we can plot the results for the intermediate case alongside the strong coupling case. For all cases, we chose $D_{s}=0$.

with the initial condition $n(z, t=0)=N_{0} \delta(z)$. Here, we defined the space fractional derivative $\partial / \partial|z|$ in the Riesz-Weyl sense whose Fourier transform takes on the simple form [25]

$$
\int_{-\infty}^{\infty} e^{i k z}\left(\frac{\partial}{\partial|z|} n(z, t)\right) d z=-|k| n(k, t)
$$

The results (4.3) and the associated dynamic equation (4.4) are quite remarkable results, which are analogous to the findings reported in Ref. [1] for a flat surface obtained from scaling arguments [substituting $x^{2}+z^{2}$ for $r^{2}$ in Eq. (6) of Ref. [1] and integrating $x$ over $(-\infty, \infty)$ produces Eq. (4.3)]. It implies that the bulk mediation causes an effective surface motion whose propagator is a Lévy stable law of index 1. This behaviour could in fact be guessed from the scaling of the returning probability to the surface, together with the diffusive scaling $z^{2} \simeq t$. However, the resulting Cauchy distribution cannot have an infinite range, as the particle in a finite time only diffuses a finite distance. The question therefore arises whether there exists a cutoff of the Cauchy law, and of what form this is.

Our exact treatment allows us to derive the Cauchy law explicitly, but also to obtain the transition to the tails of the probability density on the surface. To this end we introduce the time-dependent length scale

$$
\ell_{C}(t)=\sqrt{D_{b} t}
$$


which turns out to define the range of validity of the central Cauchy region. Thus, while at distances $z>\ell_{C}(t)$ we observe a cutoff of the Cauchy law, for $z<\ell_{C}(t)$ the Cauchy approximation is valid. Note that in the short time regime $t<t_{\kappa}$ considered here, the Cauchy range scales as $z_{\max } \approx \sqrt{D_{b} t}$ such that $z^{2}$ can indeed become significantly larger than $\kappa^{2} t^{2}$, and thus the power law asymptotics $n(z, t) \simeq z^{-2}$ in Eq. (4.3) become relevant. From the Cauchy part of the surface density we obtain the superdiffusive contribution

$$
\int_{-\ell_{C}(t)}^{\ell_{C}(t)} \frac{z^{2} \kappa t d z}{\pi\left(z^{2}+\kappa^{2} t^{2}\right)} \approx \frac{2}{\pi} \kappa \sqrt{D_{b}} t^{3 / 2}
$$

to the mean squared surface displacement. Calculation of the mean squared displacement requires, however, the limit $k \rightarrow 0$ and thus involves the extreme wings of the distribution. As the system evolves in time the central Cauchy part spreads. Already in the regime $t_{\kappa}<t<t_{a}$ we have $D_{b} t<\kappa^{2} t^{2}$, and the asymptotic behaviour $\simeq z^{-2}$ can no longer be observed. At the wings the central Cauchy distribution is truncated by Gaussian tails whose dispersion grows like $\simeq t^{3 / 2}[19]$. At longer times $t \gg t_{\kappa}$, the propagator assumes the form

$$
n(z, t) \approx \frac{N_{s}(t)}{\sqrt{4 \pi D_{b} t}} \exp \left(-\frac{z^{2}}{4 D_{b} t}\right),
$$

that is, the Gaussian shape is normalised with the the number of surface particles and the bulk diffusivity instead of the surface quantities [19].

\section{First passage statistics}

We now turn to the problem of the first passage time statistics in the present cylindrical geometry. By first passage we mean the time it takes a particle starting at some point in between the two cylinders to arrive to the inner cylinder for the first time. In analogy to our previous strategy we neglect the dependence on the azimuthal angle $\theta$ in our description. The relevant probability density for the particle position is therefore $P(r, z, t)$. Then $2 \pi r P(r, z, t) d r d z$ gives us the probability that the particle at time $t$ is in the range $(r \ldots r+d r, z \ldots z+d z)$. The initial distribution is again smeared out on a circle of radius $r_{0}$ in the plane $z=0$,

$$
\left.P(r, z, t)\right|_{t=0}=\frac{1}{2 \pi r_{0}} \delta\left(r-r_{0}\right) \delta(z) .
$$

Here the factor $1 / 2 \pi r_{0}$ appears because of the normalisation of the initial density,

$$
\left.\int_{a}^{b} r d r \int_{0}^{2 \pi} d \theta \int_{-\infty}^{\infty} d z P(r, z, t)\right|_{t=0}=1 .
$$

The time evolution of $P(r, z, t)$ is given then controlled by the ordinary diffusion equation

$$
\frac{\partial}{\partial t} P(\mathbf{r}, z, t)=D_{b} \nabla^{2} P(\mathbf{r}, z, t)
$$

valid in the radial range $a \leq r \leq b$ and on the entire cylinder axis, $-\infty<z<\infty$. The Laplace operator in azimuthal-symmetric cylindrical coordinates is

$$
\nabla^{2}=\frac{1}{r} \frac{\partial}{\partial r}\left(r \frac{\partial}{\partial r}\right)+\frac{\partial^{2}}{\partial z^{2}} .
$$

To calculate the first passage dynamics, as usual we impose an absorbing boundary condition at $r=a$ such that

$$
\left.P(r, z, t)\right|_{r=a}=0,
$$

while at the outer cylinder we keep the reflecting boundary condition

$$
\left.\frac{\partial}{\partial r} P(r, z, t)\right|_{r=b}=0 \text {. }
$$


The probability density function for the first passage time is given by the radial flux through

$$
\wp(t)=\left.2 \pi a \int_{-\infty}^{\infty} D_{b} \frac{\partial P(r, z, t)}{\partial r}\right|_{r=a} d z
$$

compare also Ref. [26]. Its Laplace transform reads

$$
\wp(s)=\left.2 \pi a D_{b} \frac{\partial P(r, k, s)}{\partial r}\right|_{r=a, k=0} .
$$

where the integral over the cylinder axis $z$ has been replaced by the zeroth Fourier mode.

\subsection{First passage time density at times $t \ll t_{b}$}

We first consider the case when the outer cylinder is remote, that is, when $t \ll t_{b}$. The first passage time density in Laplace space then reduces to [19]

$$
\wp(s)=\frac{K_{0}\left(\sqrt{s t_{0}}\right)}{K_{0}\left(\sqrt{s t_{a}}\right)},
$$

where $K_{0}$ is the zeroth-order modified Bessel function. Here, we defined the diffusion time

$$
t_{0}=\frac{r_{0}^{2}}{D_{b}}
$$

Expression (5.9) recovers a result from Ref. [12].

Expanding the Bessel functions for small arguments, we obtain

$$
\wp(s) \sim 1-2 \frac{\ln \left(r_{0} / a\right)}{\ln \left(1 /\left[s t_{a}\right]\right)} .
$$

We perform the Laplace inversion with the help of Tauberian theorems for slowly varying functions [27], leading us to the desired result

$$
\wp(t) \simeq 2 \frac{\ln \left(r_{0} / a\right)}{t \ln ^{2}\left(t / t_{a}\right)} .
$$

This expansion is valid in the range $t \gg t_{a}$. We therefore obtain a quite subtle probability density, in which the logarithm ensures normalisability, however, not even fractional moments $\left\langle t^{q}\right\rangle$ with $q>0$ exist. This extremely shallow first passage time density is characteristic for the cylindrical problem. We note that in the limit $r_{0}=a$ we recover $\wp(t)=\delta(t)$, as it should be.

\subsection{First passage time density for times $t \gg t_{b}$}

At times $t \gg t_{b}$ the outer cylinder comes into play, and we find a finite mean first passage time

$$
\langle t\rangle=\frac{b^{2}}{2 D_{b}} \ln \left(\frac{r_{0}}{a}\right),
$$

characteristic for the associated stationary regime.

\subsection{First passage time density at very short times $t \ll t_{a}$}

What is the behaviour of the first passage time density in the case of very short times, $t \ll t_{a}$ ? In this regime a particle starting close to the inner cylinder surface should not yet feel the cylindrical geometry and we expect to recover the well-known one-dimensional Lévy-Smirnov form. Starting from result (5.7), by expansion of the Bessel functions for large argument and after inverse Laplace transform we find

$$
\wp(t) \sim\left(\frac{a}{r_{0}}\right)^{1 / 2} \frac{r_{0}-a}{\sqrt{4 \pi D_{b} t^{3}}} \exp \left(-\frac{\left(r_{0}-a\right)^{2}}{4 D_{b} t}\right)\left\{1+\frac{D_{b} t}{4 a r_{0}}+\ldots\right\} .
$$


Thus, at very short times the first passage time density indeed coincides with the one-dimensional limit, reweighted by the ratio $\sqrt{a / r_{0}}$. Note that if we keep the distance $\Delta=r_{0}-a$ fixed but let both $r_{0}$ and $a$ tend to infinity, we recover the result for a flat surface,

$$
\wp_{\text {flat }}(t)=\frac{\Delta}{\sqrt{4 \pi D_{b} t^{3}}} \exp \left(-\frac{\Delta^{2}}{4 D_{b} t}\right),
$$

i.e., the well-known Lévy-Smirnov distribution.

\section{Discussion}

Bulk mediated surface diffusion (BMSD) is a ubiquitous phenomenon once for the diffusive motion of particles in the presence of a reactive boundary, i.e., when the particles may transiently bind to that surface. Cylindrical surfaces are relevant in various biological and technological situations such as BMSD of motors on microtubules, effective motion of biomolecules on the surface of cylinder-shaped bacilli, or bulk-surface dynamics along nanotubes. BMSD dynamics on cylindrical geometries may also impact technological challenges such as the colonialisation dynamics of surfaces in aqueous environments when convection is negligible, such as the spreading of bacteria or molluscs on offshore pipelines: from one mother colony new bacteria or molluscs will detach from the pipeline into the contiguous water. The spreading due to BMSD will likely relocate them to a previously uncolonised part of the pipeline surface, i.e., lead to a more efficient spreading of the creatures on the pipeline's surface.

We established an exact approach to BMSD along a reactive cylindrical surface. Our analysis reveals four distinct diffusion regimes. In all these examples it is irrelevant which specific trajectory the particles follow in the bulk, the interesting part is the effective motion on the cylinder surface. Specifically our formalism provides a stringent derivation of the transient superdiffusion discussed earlier and explicitly quantifies the transition to other regimes. Notably a saturation regime emerges for the mean squared surface displacement along the cylinder, that becomes relevant at longer times, when the particle feels the curvature of the cylinder surface $\left(t_{a}\right)$. This result mirrors a remarkable balance between the net detachment of particles into the bulk and the increasingly longer effective surface relocation when particles spend longer and longer times in the bulk. In absence of an outer confinement the saturation is indeed terminal. Only in the presence of a finite radial geometry the mean squared surface displacement returns to a linear growth in time. This observation is important for the quantitative understanding of cylindrical BMSD. In the limit $a \rightarrow \infty$ previous results for a planar surface are recovered. Relaxing the strong coupling condition an almost ballistic BMSD behaviour is found, a case that might be relevant for transport along thin cylinders such as DNA.

In Ref. [12] it was shown that the scaling behaviour in the regimes below and above $t_{a}$ can be probed experimentally by NMR methods measuring the BMSD of water molecules along imogolite nanorods over three orders of magnitude in frequency space. For larger molecules such as a protein of approximate diameter $5 \mathrm{~nm}$ we observe a diffusivity of $10^{-6} \mathrm{~cm}^{2} / \mathrm{sec}$ such that for instance the saturation plateau around a bacillus cell (radius $1 / 2 \mu \mathrm{m}$ ) sets in at around $t_{a}=2.5 \mathrm{msec}$ which might give rise to interesting consequences for the material exchange around such cells. In general, the relevance of the individual regimes will crucially depend on the scales of the surface radius and the diffusing particle (and therefore its diffusivity). It was discussed previously that even the superdiffusive short-term behaviour may become relevant $[1,5]$. In general, in a given system the separation between the various scaling regimes may not be sharp. Moreover typically a single experimental technique will not be able to probe all regimes. It is therefore vital to have available a solution for the entire BMSD problem.

Acknowledgements. Support from the Academy of Finland (FiDiPro scheme) is gratefully acknowledged.

\section{References}

[1] O. V. Bychuk, B. O'Shaughnessy. Anomalous diffusion at liquid surfaces. Phys. Rev. Lett. 74 (1995), 1795-1798. 
[2] O. V. Bychuk, B. O'Shaughnessy. Role of bulk-surface exchange in diffusion at liquid surfaces: non-Fickian relaxation kinetics. Langmuir 10 (1994), 3260-3267.

[3] J. A. Revelli, C. E. Budde, D. Prato, H. S. Wio. Bulk mediated surface diffusion: non markovian dynamics for the desorption process. New J. Phys. 7 (2005), art. no. 16.

[4] Yu. Georgievskii, E. S. Medvedev, A. A. Stuchebrukhov, Proton transport via coupled surface and bulk diffusion. J. Chem. Phys. 116 (2002), 1692; Biophys. J. 82 (2002), 2833-2846; E. S. Medvedev, A. A. Stuchebrukhov. Kinetics of proton diffusion in the regimes of fast and slow exchange between the membrane surface and bulk solution. J. Math. Biol. 52 (2006), 209-239; Proton diffusion along biological membranes. J. Phys. Cond. Mat. 23 (2011), art. no. 234103.

[5] O. V. Bychuk, B. O'Shaugnessy. Anomalous surface diffusion: a numerical study. J. Chem. Phys. 101 (1994), 772780; R. Valiullin, R. Kimmich, N. Fatkullin. Lévy walks of strong adsorbates on surfaces: Computer simulation and spin-lattice relaxation. Phys. Rev. E 56 (1997), 4371-4375.

[6] G. Oshanin, M. Tamm, O. Vasilyev. Narrow-escape times for diffusion in microdomains with a particle-surface affinity: mean-field results. J. Chem. Phys. 132 (2010), art. no. 235101.

[7] O. Bénichou, D. Grebenkov, P. Levitz, C. Loverdo, R. Voituriez. Optimal reaction time for surface-mediated diffusion. Phys. Rev. Lett. 105 (2010), art. no. 150606; Mean first passage time of surface-mediated diffusion in spherical domains. J. Stat. Phys. 142 (2011), 657-685.

[8] M. E. Toimil-Molares, L. Roentsch, W. Sigle, K.-H. Heinig, C. Trautmann, R. Neumann. Pipetting nanowires: in situ visualization of solid-state nanowire-to-nanoparticle transformation driven by surface diffusion-mediated capillarity. Adv. Funct. Mater. 22 (2012), 695-701.

[9] M. Coppey, O. Bénichou, J. Klafter, M. Moreau, G. Oshanin. Catalytic reactions with bulk-mediated excursions: Mixing fails to restore chemical equilibrium. Phys. Rev. E 69 (2004), art. no. 036115.

[10] R. Kimmich, S. Stapf, P. Callaghan, A. Coy, Microstructure of porous media probed by NMR techniques in submicrometer length scales. Magnet. Reson. Imaging 12 (1994), 339-343.

[11] S. Stapf, R. Kimmich, R.-O. Seitter. Proton and deuteron field-cycling NMR relaxometry of liquids in porous glasses: evidence for Lévy-walk statistics. Phys. Rev. Lett. 75 (1995), 2855-2858.

[12] P. Levitz, M. Zinsmeister, P. Davidson, D. Constantin, O. Poncelet. Intermittent Brownian dynamics over a rigid strand: heavily tailed relocation statistics in a simple geometry. Phys. Rev. E 78 (2008), art. no. 030102(R).

[13] M. V. Velosoa, A. G. Souza Filhoa, J. Mendes Filhoa, S. B. Faganb. Ab initio study of covalently functionalized carbon nanotubes. Chem. Phys. Lett. 430 (2006), 71-74.

[14] P. H. von Hippel, O. G. Berg. Facilitated target location in biological systems. J. Biol. Chem. 264 (1989), 675-678.

[15] I. Bonnet, A. Biebricher, P.-L. Porté. C. Loverdo, O. Bénichou, R. Voituriez, C. Escudé. W. Wende, A. Pingoud, P. Desbiolles. Sliding and jumping of single EcoRV restriction enzymes on non-cognate DNA. Nucl. Acids Res. 36,4118 (2008); I. M. Sokolov, R. Metzler, K. Pant, M. C. Williams. Target search of $N$ sliding proteins on a DNA. Biophys. J. 89 (2005), 895-902; B. v. d. Broek, M. A. Lomholt, S.-M. J. Kalisch, R. Metzler, and G. J. L. Wuite. How DNA coiling enhances target localization by proteins. Proc. Natl. Acad. Sci. USA 105 (2008), 15738-15742.

[16] Y. M. Wang, R. H. Austin, E. C. Cox. Single molecule measurements of repressor protein 1D diffusion on DNA. Phys. Rev. Lett. 97 (2006), art. no. 048302.

[17] C. Bustamante, Y. R. Chemla, N. R. Forde, D. Izhaky, Mechanical processes in biochemistry. Ann. Rev. Biochem. 73 (2004), 705-748.

[18] S. I. Coyne, N. H. Mendelson. Use of Bacillus subtilis minicells to demonstrate an antigenic relationship between the poles and lateral cylindrical regions of rod-shaped cells. Infection and Immunity 12 (1975), 1189-1194.

[19] A. V. Chechkin, I. M. Zaid, M. A. Lomholt, I. M. Sokolov, R. Metzler. Bulk-mediated surface diffusion along a cylinder: propagators and crossovers. Phys. Rev. E 79 (2009), art. no. 040105(R); Effective surface motion on a reactive cylinder of particles that perform intermittent bulk diffusion. J. Chem. Phys. 134 (2011), art. no. 204116; Bulk-mediated diffusion in a planar surface: full solution. Phys. Rev. E 86 (2012), art. no, 041101.

[20] M. A. Lomholt, I. M. Zaid, R. Metzler. Subdiffusion and weak ergodicity breaking in the presence of a reactive boundary. Phys. Rev. Lett. 98 (2007), art. no. 200603; I. M. Zaid, M. A. Lomholt, and R. Metzler, How subdiffusion changes the kinetics of binding to a surface. Biophys. J. 97 (2009), 710-721.

[21] R. B. Winter, O. G. Berg, P. H. von Hippel, Diffusion-driven mechanics of protein translocation on nucleic acids. 3. The escherichia coli lac repressor-operator interaction: kinetic measurements and conclusions. Biochem. 20 (1981), 6961-6977.

[22] E. S. Medvedev, A. A. Stuchebrukhov. Mechanism of long-range proton translocation along biological membranes. FEBS Lett. 587 (2013), 345-349.

[23] R. Metzler, J. Klafter. The random walk's guide to anomalous diffusion: a fractional dynamics approach. Phys. Rep. 339 (2000), 1-77; The restaurant at the end of the random walk: recent developments in the description of anomalous transport by fractional dynamics. J. Phys. A 37 (2004), R161-R208.

[24] A. V. Chechkin, V. Yu. Gonchar, J. Klafter, R. Metzler, L. V. Tanatarov. Lévy fights in a steep potential well. J. Stat. Phys. 115 (2004), 1505-1535.

[25] S. G. Samko, A. A. Kilbas, O. I. Marichev, Fractional Integrals and Derivatives, Theory and Applications. Gordon and Breach, New York, 1993.

[26] O. G. Berg, C. Blomberg. Association kinetics with coupled diffusional flows. Special application to the lac repressoroperator system. Biophys. Chem. 4 (1976), 367-381.

[27] S. Havlin, G. H. Weiss. A new class of long-tailed pausing time densities for the CTRW. J. Stat. Phys. 58 (1990), 1267-1273. 